\title{
Quantification of Bonded Ni Atoms for Ni-MoS 2 Metallic Contact through X-ray Photoemission Electron Microscopy
}

$\underline{\text { Xinying Shi }}^{1, *}$, Marko Huttula ${ }^{1}$, Vladimir Pankratov ${ }^{1}$, Joanna Hoszowska ${ }^{2}$, Jean-Claude Dousse ${ }^{2}$, Faisal Zeeshan $^{2}$, Yuran $\mathrm{Niu}^{3}$, Alexei Zakharov ${ }^{3}$, Zhongjia Huang ${ }^{4}$, Gang Wang ${ }^{4}$, Sergei Posysaev ${ }^{1}$, Olga Miroshnichenko $^{1}$, Matti Alatalo ${ }^{1}$, Wei Cao ${ }^{1}$

1. Nano and Molecular Systems Research Unit, University of Oulu, FI-90014, Oulu, Finland.

2. Department of Physics, University of Fribourg, Ch. du Musée 3, CH-1700 Fribourg, Switzerland.

3. MAX IV Laboratory, Lund University, P.O. Box 118, 22100, Lund, Sweden.

4. School of Mechanical and Automotive Engineering, Anhui Polytechnic University, Wuhu, China.

* Corresponding author, xinying.shi@oulu.fi

Metal-semiconductor (M/S) contacts are crucial for the performance of micro- or nano-scale electronic devices. Due to the work function difference between the metal and semiconductor as well as the Fermi level pinning effect, it is challenging to connect metal electrodes to semiconductors with good electrical contacts [1]. Conventional M/S contacts suffer from Schottky barriers, leaving high contact resistance and non-linear current-voltage relationships. Aiming at minimizing Schottky barriers, reducing contact resistance and constructing ohmic $\mathrm{M} / \mathrm{S}$ contacts, researchers have adopted various strategies, e.g., tuning surface phase states [2], inserting monolayer graphene buffer in the M/S interface [3], etc. These methods rely on sophisticated preparation processes, unavailable for wide applications.

Based on a recently proposed fabrication strategy, we successfully achieved a low resistance Ni-MoS 2 contact [4]. Featured with high hostability and tunable electronic and magnetic properties [5,6], $\mathrm{MoS}_{2}$ emerges as a promising candidate for semiconductor industry. Ni nanoparticles are bonded with $\mathrm{MoS}_{2}$ edges thanks to the Au interfacial layer. Figure 1a shows a typical contact interface, where Au atoms are present either in the form of nanocrystals or Ni-Au-MoS 2 alloy. Au atoms help to stabilize the interface structure, which has been confirmed by first-principles calculations (see Fig. 1b). While the charge transfer between $\mathrm{Ni}, \mathrm{Au}$ and $\mathrm{S}$ atoms is responsible for chemical bonding at the interface, the amount of $\mathrm{Ni}$ atoms participating in the bonding to the host is not known. Spectroscopic determinations at nanoregions are therefore needed to distinguish chemically bonded guest $\mathrm{Ni}$ and evaluate its quantity.

Here we report a detailed quantification work on determining effective Ni atoms involved in the bonding formation. Employing synchrotron radiation based X-ray photoemission electron microscopy (XPEEM), we are able to focus on selected nano-regions and collect local X-ray absorption spectra (XAS). To compensate for the relatively low spatial resolution of the XPEEM, scanning electron microscopy (SEM) investigation was carried out first (Fig. 2a). Two interfaces marked with dashed rectangles were studied. In each of the interfaces, a sequence of acentric circular regions of Ni nanoparticles with diameters of 25, 50, 75, 100 and $150 \mathrm{~nm}$ were probed within XPEEM field of view (Fig. 2b). Here we use the distance to interface to describe these nano-regions with certain diameters. With increasing distance, more $\mathrm{Ni}$ atoms far from the Ni-MoS 2 interface are included.

XAS spectra of Ni $2 p \mathrm{~L}_{2,3}$ edges were collected with photon energies from $845 \mathrm{eV}$ to $875 \mathrm{eV}$. The inset in Fig. 2c shows the spectrum obtained at interface 1 with a distance of $50 \mathrm{~nm}$. Ni $2 p_{3 / 2}$ and $2 p_{1 / 2}$ features are identified corresponding to the $2 p^{6} 3 d^{8} \rightarrow 2 p^{5} 3 d^{9}$ transition, and both of them split into two components. In addition, the peak at $\sim 861 \mathrm{eV}$ is attributed to Ni-S bonding in confined spaces, different 
from previously reported $\mathrm{Ni}$ satellite peaks [7]. Thus the ratio of this peak to the $2 p_{3 / 2}$ feature denotes the percentage of Ni atoms involved in bonding formation, as shown in Fig. 2c. Within the distance of 25 $\mathrm{nm}, 50 \% \sim 60 \%$ of $\mathrm{Ni}$ atoms participate in bonding with adjacent foreign atoms. The percentage falls with larger distance to interface. The absolute number of direct bonded $\mathrm{Ni}$ atoms is nearly constant with a distance over $100 \mathrm{~nm}$, suggesting a possible threshold dimension of the $\mathrm{Ni}-\mathrm{MoS}_{2}$ interface.

This work takes advantage of the XPEEM technique and provides a feasible route to evaluate the quality of a M/S contact interface by examining the amount of metal atoms participating in chemical bonding. It also helps for selecting metal electrodes for a specific semiconductor device [8].

References:

[1] A. Allain et al, Nat. Mater. 14 (2015) p. 1195.

[2] R. Kappera et al, Nat. Mater. 13 (2014) p.1128.

[3] W. S. Leong et al, ACS Nano 9 (2015) p. 869.

[4] X. Shi et al, Small 14 (2018) 1704526.

[5] W. Cao et al, Mater. Chem. Phys. 158 (2015) p. 89.

[6] X. Shi et al, Crystals 8 (2018) 24.

[7] T. Kroll et al, J. Chem. Phys. 137 (2012) 054306.

[8] The authors acknowledge funding from the European Commission's Seventh Framework Programme (FP7/2007-2013) CALIPSO (Grant No. 312284). X.S. thanks the scholarship sponsored by China Scholarship Council. J.H., J.-Cl.D. and F.Z. acknowledge the financial support of the Swiss National Science Foundation (Grant No. 200020_146739).
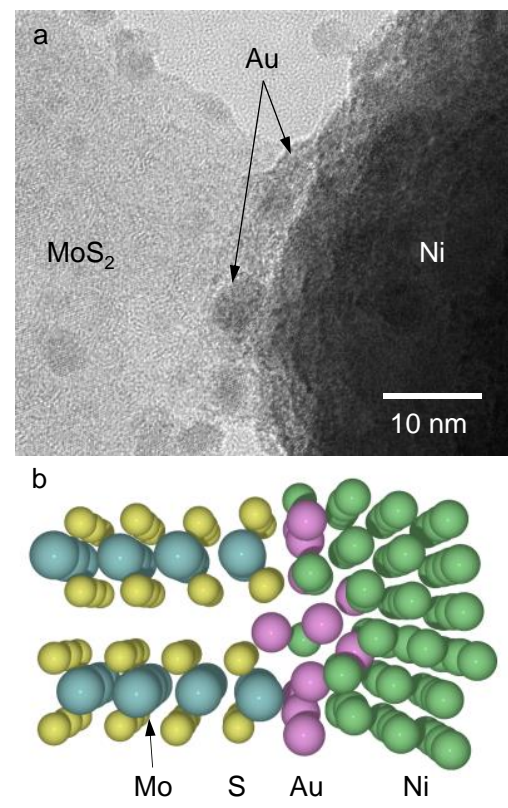

Figure 1. (a) HRTEM image shows the morphology of $\mathrm{Ni}-\mathrm{Au}-\mathrm{MoS}_{2}$ interface. (b) Optimized structure of $\mathrm{Mo}_{24} \mathrm{~S}_{48} \mathrm{Au}_{12} \mathrm{Ni}_{72}$ supercell.
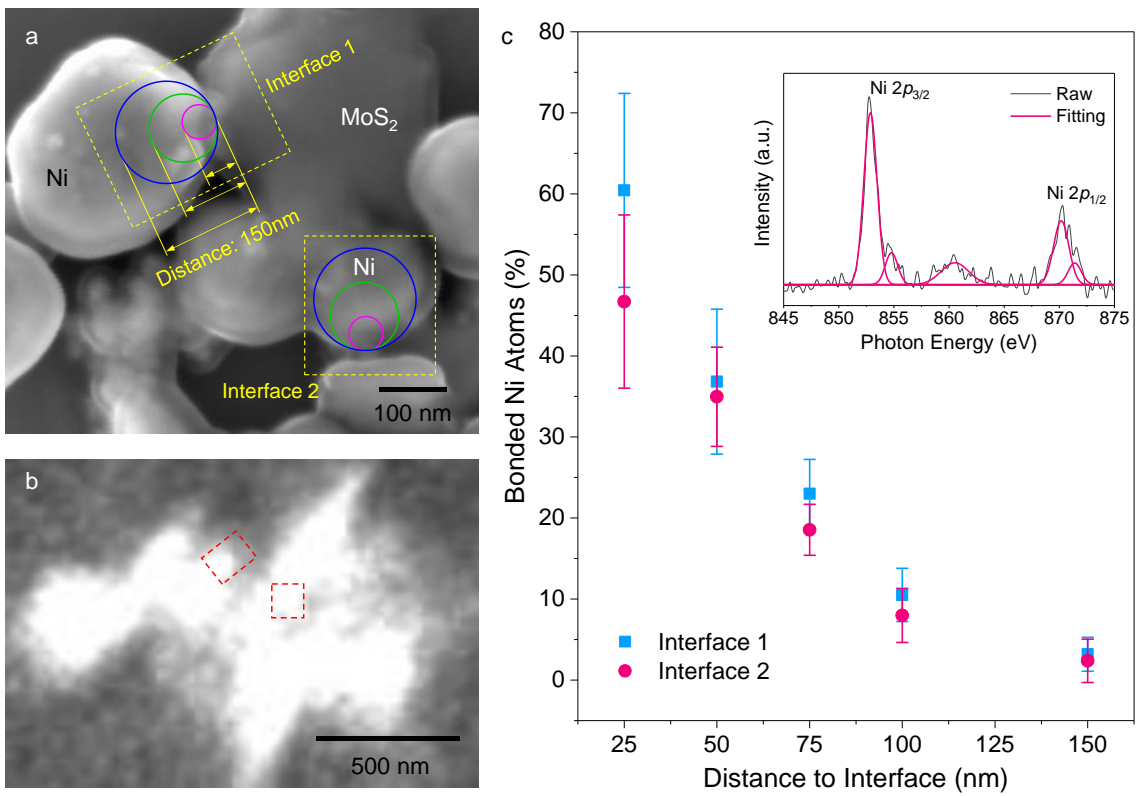

Figure 2. (a) SEM image of Ni-Au-MoS 2 composite. (b) XPEEM view. The two Ni-MoS 2 interfaces in (a) are also marked here with dashed rectangles. (c) Calculated percentage of bonded $\mathrm{Ni}$ atoms. The inset shows XAS spectra of Ni $2 p \mathrm{~L}_{2,3}$ edges obtained within a distance of $50 \mathrm{~nm}$ to the interface. 